\title{
Nationwide Survey on Actual Interventions for Type 2 Diabetes by Japanese Practitioners (NSAID Study-1): Glycemic, Weight, and Blood Pressure Management
}

\author{
Keiko Arai (D) - Tetsuo Nishikawa - Shohei Yuasa · Shin-Ichiro Shirabe • \\ Yoko Matsuzawa $\cdot$ Shigeyuki Ohtsu $\cdot$ Koich Hirao · Hisao Mori
}

Received: April 16, 2020 / Published online: May 22, 2020

(C) The Author(s) 2020

\section{ABSTRACT}

Introduction: Considering the increase in the number of patients with diabetes, the quality of diabetes care provided by general practitioners (GP) is critical for preventing complications. We

Digital Features To view digital features for this article go to https://doi.org/10.6084/m9.figshare.12264728.

K. Arai $(\bowtie)$

Arai Clinic, Yokohama, Japan

e-mail: arai-cl@n04.itscom.net

T. Nishikawa

Nisikawa Clinic, Yokohama, Japan

S. Yuasa

Syohei Clinic, Kamakura, Japan

S.-I. Shirabe · K. Hirao

Health Education Center Science Clinic, Yokohama, Japan

Y. Matsuzawa

Matsuzawa Diabetes Clinic, Yokohama, Japan

S. Ohtsu

Nakanoshima Diabetes Clinic, Kawasaki, Japan

H. Mori

Yokohama Sotetsu Building Clinic of Internal

Medicine, Yokohama, Japan

K. Arai - T. Nishikawa $\cdot$ S. Yuasa - S.-I. Shirabe

Y. Matsuzawa · S. Ohtsu · K. Hirao · H. Mori

Department of Clinical Research of Kanagawa

Association of Medical and Dental Practitioners,

Yokohama, Japan performed a nationwide survey to determine whether the diabetic management provided to patients with type 2 diabetes mellitus (T2DM) by Japanese practitioners is appropriate.

Methods: We randomly selected 463 clinics throughout Japan; 8070 patients with T2DM (6525 and 1545 under the care of GP and specialists $[\mathrm{SP}]$, respectively) were enrolled. We obtained information on hemoglobin A1c (HbA1c) levels, age, height, body weight, diabetes type and treatment modality, blood pressure (BP), and hypertension or dyslipidemia from each patient. Additionally, we surveyed the collaborations among physicians.

Results: The median HbA1c level of patients treated by GP was lower than that of patients treated by SP (6.8 [6.2-7.3], median [interquartile range] vs. 6.9 [6.5-7.5], $p<0.0001)$. The percentage of patients receiving insulin therapy was also higher (23.8\%) among patients treated by SP than among those treated by GP (8.6\%). Patients not receiving insulin therapy showed lower median HbA1c levels than those receiving insulin therapy, irrespective of the care provider. The mean body mass index of patients with HbA1c levels $<6.9 \%$ or $>9.0 \%$ cared for by SP was lower than that of those cared for by GP. The rate of target $\mathrm{BP} \quad(<140 / 90 \mathrm{mmHg})$ achievement was $73.2 \%$ and $73.3 \%$ among patients with T2DM and hypertension cared for by GP and SP, respectively. Furthermore, $88.2 \%$ of GP reported that consulting with SP was easy. 
Conclusion: The present study clearly demonstrated that many patients with T2DM are appropriately cared for by general practitioners instead of diabetes specialists in Japan, although the number of diabetes specialists is insufficient to cover all patients with diabetes.

Keywords: Blood pressure; Body weight; Collaboration; General practitioners; Glycemic control; Nationwide survey; Type 2 diabetes mellitus

\section{Key Summary Points}

Why carry out this study?

The number of patients with diabetes and the associated national medical healthcare expenditures are still increasing in Japan

The quality of diabetic management by general practitioners is critical to reduce diabetes complications, medical costs, and improve patient quality of life instead of diabetes specialists, although the number of specialists is insufficient to cover all patients with diabetes in Japan

\section{What was learned from the study?}

Many patients with type 2 diabetes mellitus are appropriately cared for by general practitioners, e.g., in terms of glycemic, weight, and blood pressure control, instead of by diabetes specialists in Japan

The collaboration between general practitioners and specialists may be appropriate for managing patients with diabetes in Japan

To match the constant increase in demand for diabetes care, providing adequate quality of care by general practitioners is a solution to decrease diabetic complications and improve the quality of life for patients with diabetes

\section{INTRODUCTION}

The goal in the treatment of patients with diabetes mellitus is to prevent disease complications. This leads to a prolonged life span, improved quality of life regardless of diabetes, and reduced medical costs. The number of patients with diabetes and the associated national medical healthcare expenditures are still increasing in Japan [1]. The annual Health, Labour and Welfare Report of 2017 showed that approximately 9.5 and 11 million persons (7.9\% and $9.1 \%$ of total population in Japan) were strongly suspected to or considered to have diabetes, respectively [1].

Some European countries have a universal healthcare structure based on a gate-keeping system. In this system, patients visit primary care physicians first after obtaining an appointment, and then primary care physicians refer them to specialists if necessary. Japan has a public health insurance system for everyone in the country. This Japanese medical service is based on a free-access policy. Every person in Japan can obtain medical services at any clinic or hospital at any time. In addition, Japanese physicians have the freedom of opening clinics or hospitals specializing in any area. Under these circumstances, patients with type 2 diabetes mellitus (T2DM) can choose to visit either diabetes specialists or general practitioners depending on their preference. Moreover, approximately 6000 physicians in Japan have been certified as specialists for diabetes care by the Japan Diabetes Society (JDS). This number of diabetes specialists is insufficient to cover all patients with diabetes, and as a result, general practitioners care for many patients with diabetes in Japan. Therefore, the quality of diabetic management by general practitioners as well as by diabetes specialists is critical to reduce diabetes complications, improve quality of life, and reduce medical costs.

To estimate the quality of diabetic management in this study, we selected hemoglobin A1c (HbA1c) levels as a surrogate marker of glycemic control, and body weight and blood pressure control as other outcome measures. Glycemic control remains a major therapeutic objective, 
and measurement of HbA1c levels is now accepted as a standard index of glycemic control in patients with diabetes. In fact, HbA1c levels are also a major outcome measure and have been used as the standard in the Diabetes Control and Complication Trial and other studies [2].

Meanwhile, weight management and reduction are critical for patients with diabetes, and sustained weight loss is associated with low HbA1c levels [3]. Weight reduction for patients with diabetes requires lifestyle interventions in addition to medication assistance. For empowering patients to change their lifestyle, many co-medical staff, such as dietitians, exercise trainers, and psychologists, are required to be collaborate, while every general practitioner may not hire or collaborate with these special staff. Data from the Japan Diabetes Clinical Data Management Study Group (JDDM) that consisted of diabetes specialists in Japan showed that the yearly change in mean body mass index (BMI) increased until 2013 when weightlowering drugs such as sodium-glucose cotransporter 2 inhibitors (SGLT2i) and glucagon-like peptide 1 receptor agonists (GLP-1 RA) became available [4]. These findings suggest that weight management for patients with T2DM may not be easy for general practitioners.

Hypertension is a major risk factor for atherosclerotic cardiovascular disease, heart failure, and microvascular complications [5], and many studies have demonstrated the clinical efficiency of blood pressure reduction in preventing these complications. Blood pressure is routinely measured at every visit in a realworld clinical setting. Therefore, we used the blood pressure assessed at the clinic as one of the outcome measures of this study.

We performed a nationwide cross-sectional survey in 2006 and reported that the average HbA1c levels in Japanese patients treated by both general practitioners and specialists were acceptable [6]. In Japan, seven classes of oral antidiabetic drugs (OADs) such as biguanides (BG), thiazolidinediones (TZD), sulfonylureas (SU), rapid-acting insulin secretagogues (glinides), dipeptidyl peptidase 4 inhibitors (DPP4i), $\alpha$-glucosidase inhibitors $(\alpha-G I)$, and SGLT2i are available now. Among these OADs, DPP4i and
SGLT2i became available recently. The risk of hypoglycemia of drugs in these two classes is less than that with drugs that stimulate insulin secretion such as SU and glinides. In addition, Japanese guidelines for elderly patients with diabetes have shifted from strict glycemic control to the avoidance of hypoglycemia [7]. As trends in prescriptions have changed [8], in 2018 we performed a survey by collecting questionnaires from practitioners throughout Japan to clarify whether the glycemic, body weight, and blood pressure management provided to patients with T2DM by general practitioners is appropriate instead of diabetes specialists under the situation of insufficient specialists to cover all patients with diabetes in Japan.

\section{METHODS}

\section{Study Design}

This study was a nationwide cross-sectional survey of 8070 patients with T2DM who were cared for by 463 practitioners.

\section{Ethical Considerations}

The present study was approved by the ethics committee of the Kanagawa Association of Medical and Dental Practitioners, which includes lawyers and ethical experts, on May 1, 2018. The study approval number was 17007 . Informed consent was obtained from all enrolled patients at each clinic, in accordance with the Guidelines for Epidemiological Study of the Ministry of Health, Labour and Welfare of Japan.

\section{Patients and Methods}

We randomly selected 6580 practitioners (approximately $15 \%$ ) who were members of the Japanese Medical and Dental Practitioners for the Improvement of Medical Care (JMDPIMC) group and asked them to participate in this study. A total of 463 practitioners agreed to participate, and 8070 patients with T2DM who 
were 33-100 years old were enrolled. The type of diabetes mellitus was determined according to the criteria described in the "Report of the Committee of Japan Diabetes Society (JDS) on the Classification and Diagnostic Criteria of Diabetes Mellitus" [9]. These criteria are almost identical to those of the World Health Organization [10]. Briefly, type 1 diabetes was diagnosed on the basis of permanent insulinopenia and the state of being ketosis-prone (idiopathic type 1 diabetes) or on the basis of positivity for autoimmune destruction markers such as glutamic acid decarboxylase (immune-mediated type 1 diabetes).

A diabetes specialist $(n=79)$ in this study was defined as a member or board-certified diabetes care physician from the JDS or Japan Endocrine Society. Other physicians $(n=384)$ were considered general practitioners.

Data were collected from July 1 to July 31, 2018. Inclusion criteria required subjects to visit a practitioner and have their HbA1c levels tested at least once every 3 months. Each practitioner was encouraged to enroll up to 20 patients in order of arrival. Patient age, sex, height, body weight, most recent HbA1c data, blood pressure, hypertension or dyslipidemia status, and details of therapy including drugs and insulin were collected for analysis. Weight and height were measured using standardized techniques and equipment. BMI was calculated as the weight in kilograms divided by the square of the height in meters. In addition, we asked practitioners for their age, sex, clinic location, reasons for consulting with a diabetes specialist or being consulted by a general practitioner, and information for decision-making for prescriptions. Data were filled out in a sheet by each practitioner and collected by mail at the central analytical facility, where the information was treated anonymously.

\section{Statistical Analysis}

The data distribution was analyzed using Kolmogorov-Smirnov test and Shapiro-Wilk test. Levels of HbA1c, age, BMI, and blood pressure are presented as the median with interquartile range, as appropriate, according to the data distribution. Differences between two groups were analyzed using a Mann-Whitney $U$ test. The Kruskal-Wallis test was applied for multiple comparisons. The chi-square test was used to compare the distributions of HbA1c, treatment modality, and rate of target blood pressure achievement between groups. All statistical analyses were performed using the statistical software package SPSS (SPSS Inc., Chicago, IL, USA) and JMP version 14.0 software (SAS Institute Inc., NC, USA). $p$ values less than 0.05 were considered statistically significant.

\section{RESULTS}

\section{Characteristics of Study Population}

A total of 8070 patients with T2DM were enrolled in the study. Of these, 6525 (80.9\%) and 1545 (19.1\%) patients were cared for by general practitioners and diabetes specialists, respectively. The clinical characteristics of the patients are summarized in Table 1 . The age, ratio of women to men, BMI, and prevalence of hypertension among patients cared for by general practitioners were higher than those among patients cared for by diabetes specialists, whereas the prevalence of hyper-low-density lipoprotein (LDL) cholesterolemia was not different between the two groups $(53.0 \%$ and $51.3 \%$, respectively, $p=0.251$ ). In addition, there was no significant difference in the prescription rate of statins for patients with T2DM and hyper-LDL cholesterolemia between general practitioners and specialists $(84.1 \%$ and $84.3 \%$, respectively, $p=0.874$ ).

\section{HbA1c Values by Age and BMI Group Between Two Care-Provider Categories}

The median HbA1c level of patients treated by general practitioners was lower than that of patients treated by diabetes specialists $(6.8 \%$ [6.2-7.3], median [interquartile] vs. $6.9 \%$ [6.5-7.5], $p<0.0001$ ) (Fig. 1a); this observation was true irrespective of whether the patients were divided according to age $(<65, \geq 65$ and 
Table 1 Characteristics of the patients

\begin{tabular}{lllr}
\hline & General practitioners $(\boldsymbol{n}=384)$ & Specialists $(\boldsymbol{n}=79)$ & $\boldsymbol{p}$ \\
\hline Number $(n, \%)$ & $6525(80.9 \%)$ & $1545(19.1 \%)$ & $<0.001$ \\
Age (years old) & $71(64-79)$ & $70(61-77)$ & $<0.001$ \\
Sex $($ male/female, $\%)$ & $55.0: 45.0$ & $60.0: 40.0$ & 0.001 \\
BMI $\left(\mathrm{kg} / \mathrm{m}^{2}\right)$ & $24.5(22.2-27.2)$ & $24.2(21.7-27.0)$ & $<0.001$ \\
Hypertension $(n, \%)$ & $4638(71.0 \%)$ & $938(61.0 \%)$ & 0.251 \\
Hyper-LDL cholesterolemia $(n, \%)$ & $3441(53.0 \%)$ & $790(51.3 \%)$ & 0.874 \\
Treatment with statin & $84.1 \%$ & $84.3 \%$ & \\
\hline
\end{tabular}

Data of age and BMI are presented as median (interquartile range)

$B M I$ body mass index, $L D L$ low-density lipoprotein

(a)



Fig. 1 Median HbAlc levels and distribution for all patients. a Median HbAlc level for all patients treated by general practitioners was lower than that of those treated by diabetes specialists $\left({ }^{*} p<0.0001\right)$. The bottom of the boxplot indicates the 25th percentile, and the top indicates the 75 th percentile. Horizontal lines in the boxes represent

$<75, \geq 75$ years $)$ or BMI $(<22, \geq 22$ and $<25$, $\geq 25$ and $<27, \geq 27$ ) (Table 2).

Multivariable logistic regression analysis also showed that a lower age and a higher BMI were (b)

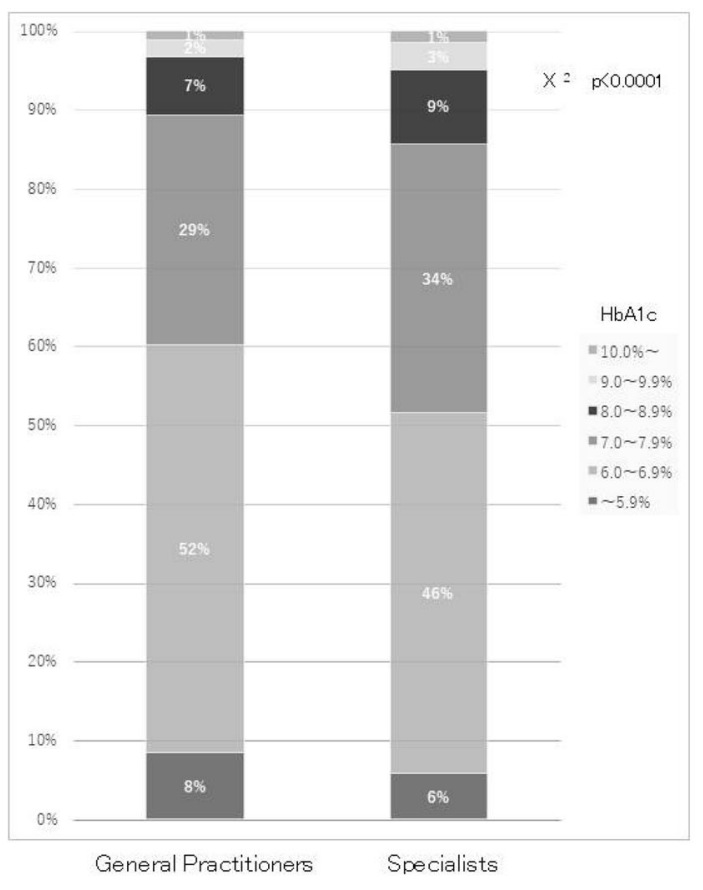

medians. The lower and upper ends of the whiskers represent the minimum and maximum observations, respectively. b Distributions of patients according to $\mathrm{HbAlc}$ level rank were also different between patients treated by general practitioners and those treated by diabetes specialists (chi-square test, $p<0.0001$ )

associated with higher HbA1c values $(p<0.0001)$ (Table 3). 
Table $2 \mathrm{HbA1c}$ values by age and BMI groups

\begin{tabular}{lllr}
\hline & General practitioners & Specialists & $p$ \\
\hline Age $($ years old $)$ & & & $<0.0001$ \\
$<65$ & $6.9 \%(6.4-7.5)$ & $7.0 \%(6.5-7.6)$ & 0.006 \\
$\geq 65$ and $<75$ & $6.7 \%(6.3-7.2)$ & $6.9 \%(6.5-7.5)$ & $<0.0001$ \\
$\geq 75$ & $6.7 \%(6.3-7.2)$ & $6.9 \%(6.6-7.5)$ & 0.006 \\
BMI $\left(\mathrm{kg} / \mathrm{m}^{2}\right)$ & & & $<0.0001$ \\
$<22$ & $6.7 \%(6.2-7.2)$ & $6.8 \%(6.3-7.4)$ & 0.001 \\
$\geq 22$ and $<25$ & $6.7 \%(6.3-7.3)$ & $6.9 \%(6.5-7.5)$ & $<0.0001$ \\
$\geq 25$ and $<27$ & $6.8 \%(6.4-7.3)$ & $6.9 \%(6.5-7.5)$ & $7.0 \%(6.6-7.6)$ \\
$\geq 27$ & $6.8 \%(6.4-7.4)$ & & \\
\hline
\end{tabular}

Data of HbAlc values are presented as median (interquartile range)

$B M I$ body mass index

\section{Blood Pressure Control at Doctor's Office}

The median office systolic blood pressure of patients with T2DM and hypertension who were cared for by general practitioners and those who were cared for by specialists was not different (130.0 mmHg [90.0-209.0] and $130.0 \mathrm{mmHg}$ [86.0-194.0], respectively, $p=0.296$ ), whereas the median diastolic blood pressure of patients cared for by general practitioners was $1 \mathrm{mmHg}$ higher than that of patients cared for by specialists $(71.0 \mathrm{mmHg}$
[38.0-118.0]) vs. $70.0 \mathrm{mmHg} \quad$ [40.0-125.0], respectively, $p<0.001$ ) (Table 4$)$. The achievement rates of target blood pressure $<140$ / $90 \mathrm{mmHg}$ and $<130 / 80 \mathrm{mmHg}$ in patients with T2DM and hypertension cared for by general practitioners and specialists were not different $(73.2 \%$ and $73.3 \%$ for $<140 / 90 \mathrm{mmHg}$, $p=0.968 ; \quad 37.7 \%$ and $40.0 \%$ for $<130 /$ $80 \mathrm{mmHg}, p=0.182$, respectively) (Table 4 ). An angiotensin-converting enzyme inhibitors (ACEi) or angiotensin receptor blockers (ARB) was less commonly prescribed for patients with

Table 3 Contributing factors for HbAlc levels by multivariable regression analysis

\begin{tabular}{llcc}
\hline & Unadjusted $\boldsymbol{\beta}$ coefficient $(\mathrm{SE})$ & Adjusted $\boldsymbol{\beta}$ coefficient & $\boldsymbol{p}$ \\
\hline Number of OADs & $0.012(0.001)$ & 0.242 & $<0.0001$ \\
With insulin therapy & $0.41(0.02)$ & 0.245 & $<0.0001$ \\
Age & $0.000(0.000)$ & -0.052 & $<0.0001$ \\
BMI & $0.001(0.000)$ & 0.059 & $<0.0001$ \\
Sex as female & $-0.003(0.001)$ & -0.031 & 0.004 \\
Specialists as a care provider & $0.03(0.001)$ & 0.024 & 0.025 \\
\hline
\end{tabular}

$S E$ standard error, $O A D s$ oral antidiabetic drugs (including biguanides, thiazolidinediones, sulfonylureas, rapid-acting insulin secretagogues, dipeptidyl peptidase 4 inhibitors, $\alpha$-glucosidase inhibitors, sodium-glucose cotransporter 2 inhibitors), $B M I$ body mass index 
Table 4 Blood pressure control at doctor's office

\begin{tabular}{lllr}
\hline & General practitioners & Specialists & $p$ \\
\hline Patients without hypertension & & & \\
Systolic blood pressure $(\mathrm{mmHg})$ & $124.0(78.0-169.0)$ & $124.0(83.0-171.0)$ & 0.231 \\
Diastolic blood pressure $(\mathrm{mmHg})$ & $70.0(45.0-106.0)$ & $71.0(39.0-114.0)$ & 0.020 \\
Patients with hypertension & & & \\
Systolic blood pressure $(\mathrm{mmHg})$ & $130.0(90.0-209.0)$ & $130.0(86.0-194.0)$ & 0.296 \\
Diastolic blood pressure $(\mathrm{mmHg})$ & $71.0(38.0-118.0)$ & $70.0(40.0-125.0)$ & $<0.001$ \\
Achievement rate $<140 / 90 \mathrm{mmHg}$ & $73.2 \%$ & $73.3 \%$ & 0.968 \\
Achievement rate $<130 / 80 \mathrm{mmHg}$ & $37.7 \%$ & $40.0 \%$ & 0.182 \\
Treatment with ACEi/ARB & $68.3 \%$ & $72.3 \%$ & 0.016 \\
Treatment with CCB & $68.6 \%$ & $63.9 \%$ & 0.005 \\
\hline
\end{tabular}

$A C E i$ angiotensin-converting enzyme inhibitors, $A R B$ angiotensin receptor blockers, $C C B$ calcium channel blockers

T2DM and hypertension cared for by general practitioners than for those cared for by specialists $(68.3 \%$ vs. $72.3 \%$, respectively, $p=0.016)$, whereas a calcium channel blockers (CCB) was more frequently prescribed by general practitioners than by specialists $(68.6 \%$ vs. $63.9 \%$, respectively, $p=0.005$ ) (Table 4 ).

\section{BMI by Age and HbA1c Level Between Two Care-Provider Categories}

We evaluated the weight control of the patients according to HbA1c level, age, and whether care was provided by a general practitioner or diabetes specialist. Among patients of all age groups, the median BMI of patients with $\mathrm{HbA1c}$ levels $<6.9 \%$ and $>9.0 \%$ cared for by specialists was lower (23.7 [21.4-26.6] and 24.6 [22.7-27.5], respectively) than that of those cared for by general practitioners (24.3 [22.1-26.8] and 26.0 [23.0-29.3], respectively, $p<0.05$ ) (Fig. 2). Among patients aged $<65$ and $>75$ years, the BMI of patients with an HbA1c level $<6.9 \%$ cared for by specialists was lower (25.2 [22.6-28.5] and 22.7 [20.6-25.2]) than that of those cared for by general practitioners (25.9 [23.2-29.1] and 23.7 [21.6-29.1], $p<0.05)$. Finally, in patients aged $65-75$ years, the BMI of patients with an HbA1c level $>9.0 \%$ cared for by specialists was lower (24.5 [23.2-26.4]) than that of those cared for by a general practitioner (26.1 [24.4-28.4], $p<0.05$ ) (Fig. 2).

\section{Distribution of HbA1c Levels}

The distribution of patients according to the range of HbA1c levels was also different between general practitioners and specialists (chi-square test, $p<0.0001$ ) (Fig. 1b). The proportions of patients with HbA1c levels $<7.0 \%$ treated by general practitioners and specialists were $60 \%$ and $52 \%$, respectively, whereas those of patients with HbA1c levels $>8.0 \%$ treated by general practitioners and specialists were 10\% and $13 \%$, respectively.

\section{Differences in HbA1c Values According to Type of Therapy}

The proportions of each therapy type are listed in Table 5. There was a difference between general practitioners and specialists (chi-square test, $p<0.0001$ ). The percentages of patients treated with OADs by general practitioners and 
(a) All age

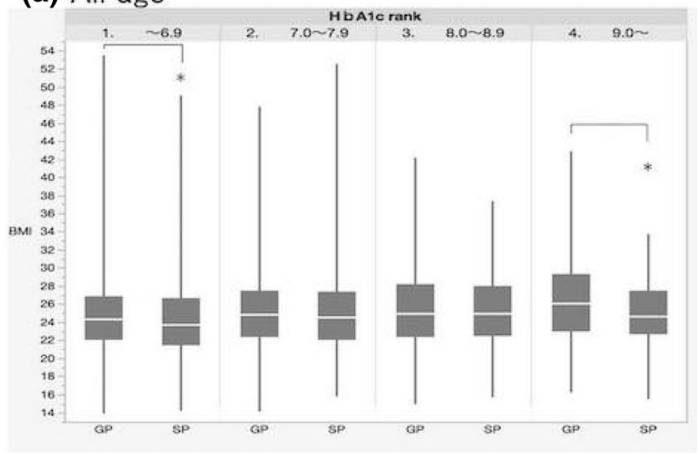

(c) Age $65-75$ y.o.

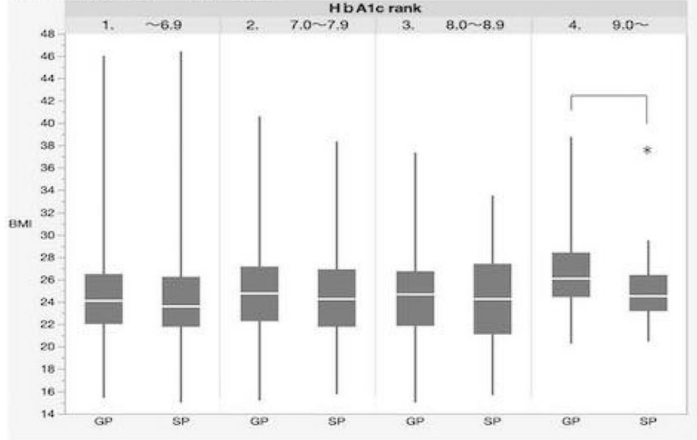

Fig. 2 BMI of patients cared for by general practitioners or specialists according to HbAlc rank. a Patients of all ages, $\mathbf{b}$ patients aged $<65$ years, $\mathbf{c}$ patients aged $65-75$ years, and $\mathbf{d}$ patients aged $>75$ years. GP general practitioners, SP specialists, ${ }^{*} p<0.05$. The bottom of the

specialists were $84.5 \%$ and $68.5 \%$, respectively. In OAD therapy, the number of different OADs prescribed for patients treated by general practitioners was lower than that for patients treated by specialists $(2[1,2]$ vs. 2 [1-3]; $p<0.0001$, Mann-Whitney $U$ test).

The percentages of patients receiving insulin therapy with or without other drugs were $8.6 \%$ and $23.8 \%$ for those treated by general practitioners and specialists, respectively. The percentages of patients treated with a GLP-1 RA with or without other drugs were $1.9 \%$ and $5.5 \%$ for those treated by general practitioners and specialists, respectively.

The median HbA1c levels among patients treated with OADs were higher than those among patients treated with only diet therapy by either care provider (Table 6). In addition, (b) Age $<65$ y.o.

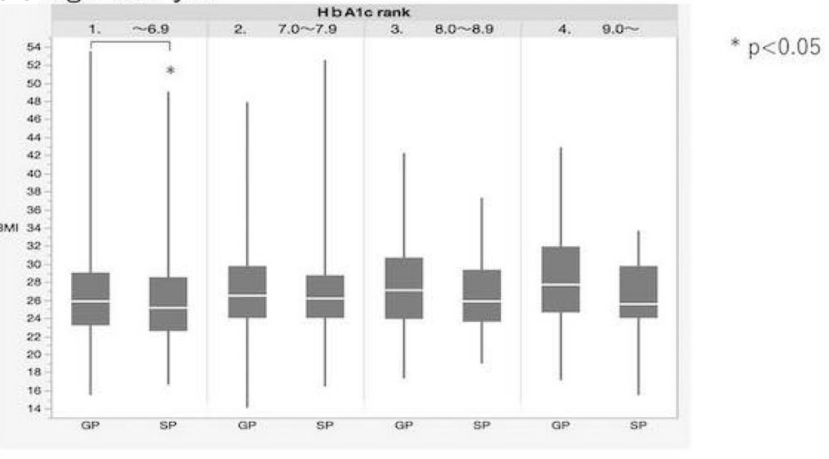

(d) Age $\geq 75$ y.o.

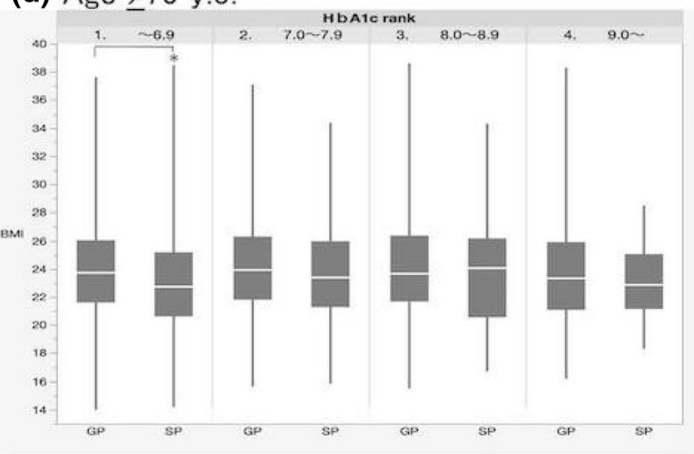

boxplot indicates the 25 th percentile, and the top indicates the 75th percentile. Horizontal lines in the boxes represent medians. The lower end of the whiskers represents the minimum observation, and the upper end represents the maximum

the median HbA1c levels for patients treated with insulin, insulin plus OADs, GLP-1 RA plus OADs, and insulin plus GLP-1 RA plus OADs were higher than those for patients treated with only diet therapy or only OADs by either general practitioners or specialists (Table 6). There were no significant differences in the median HbA1c levels of patients treated with any type of therapy, except for those treated with only OADs, by general practitioners or specialists. With regard to OAD therapy, the median HbA1c levels of patients treated by general practitioners were lower than those of patients treated by specialists (6.7 [6.3-7.3] vs. $6.8 \%$ [6.4-7.3], respectively, $p=0.001)$. A multivariable model showed that higher HbA1c levels were associated with a higher number of prescribed OADs (Table 3). 
Table 5 Proportion of the therapy by each care provider

\begin{tabular}{lcr}
\hline Type of therapy & $\begin{array}{l}\text { General practitioners } \\
\boldsymbol{n}(\%)\end{array}$ & $\begin{array}{l}\text { Specialists } \\
\boldsymbol{n}(\%)\end{array}$ \\
\hline Diet & $461(7.1 \%)$ & $75(4.9 \%)$ \\
OADs & $5423(83.1 \%)$ & $1058(68.5 \%)$ \\
GLP-1 RA & $11(0.2 \%)$ & $4(0.3 \%)$ \\
Insulin & $116(1.8 \%)$ & $85(5.5 \%)$ \\
GLP-1 RA + OADs & $73(1.1 \%)$ & $53(3.4 \%)$ \\
Insulin + OAD & $402(6.2 \%)$ & $242(15.7 \%)$ \\
Insulin + GLP-1 RA & $15(0.2 \%)$ & $5(0.3 \%)$ \\
Insulin + GLP-1 RA + OADs & $25(0.4 \%)$ & $23(1.5 \%)$ \\
Total & $6526(100 \%)$ & $1545(100 \%)$ \\
\hline
\end{tabular}

$\left(\chi^{2}\right.$ test, $\left.p<0.0001\right)$

$O A D s$ oral antidiabetic drugs (including biguanides, thiazolidinediones, sulfonylureas, rapid-acting insulin secretagogues, dipeptidyl peptidase 4 inhibitors, $\boldsymbol{\alpha}$-glucosidase inhibitors, sodium-glucose cotransporter 2 inhibitors), GLP-1 RA glucagonlike peptide 1 receptor antagonisits

Table 6 Difference of HbAlc values by the type of therapy

\begin{tabular}{llll}
\hline Type of therapy & General practitioners & Specialists & $p$ \\
\hline Diet & $6.5(6.2-6.8)$ & $6.4(6.1-8.8)$ & 0.280 \\
OADs & $6.7(6.3-7.3)^{*}$ & $6.8(6.4-7.3)^{*}$ & 0.001 \\
GLP-1 RA & $6.6(6.1-7.9)$ & $6.5(6.3-7.5)$ & 0.948 \\
Insulin & $7.3(6.6-8.1)^{* *}$ & $7.4(6.9-8.0)^{* \#}$ & 0.617 \\
GLP-1 RA + OADs & $7.2(6.8-8.0)^{* \#}$ & $7.4(7.0-8.5)^{* \#}$ & 0.384 \\
Insulin + OADs & $7.3(6.8-8.0)^{* \#}$ & $7.4(6.8-8.0)^{* \#}$ & 0.091 \\
Insulin + GLP-1 RA & $7.1(6.6-7.6)$ & $7.3(6.1-8.5)$ & 0.745 \\
Insulin + GLP-1 RA + OADs & $7.3(6.8-8.3)^{* \$}$ & $7.4(7.2-7.7)^{* \$}$ & 0.652 \\
\hline
\end{tabular}

$O A D s$ oral antidiabetic drugs (including biguanides, thiazolidinediones, sulfonylureas, rapid-acting insulin secretagogues, dipeptidyl peptidase 4 inhibitors, $\boldsymbol{\alpha}$-glucosidase inhibitors, SGLT2 inhibitors), GLP-1 RA glucagon-like peptide 1 receptor antagonists

Data of $\mathrm{HbAlc}$ are presented as median (interquartile range)

${ }_{\#}^{*} p<0.0001$ vs. diet by each care provider

${ }^{\#} p<0.0001$ vs. OADs by each care provider

$\$ p<0.001$ vs. OADs by each care provider 


\section{Consultation Between General Practitioners and Specialists}

In response to the question on whether consulting with specialists was easy, $88.2 \%$ of general practitioners replied that it was easy. The reasons for consultation are summarized in Table 7. The most frequent reason for the consultation was the initiation of insulin; the second most frequent reason was a high $\mathrm{HbA1c}$ level. However, the HbA1c level that general practitioners wished to consult with specialists for was higher than that for which specialists preferred to be consulted $(9.5 \pm 1.4 \%$, mean \pm SD vs. $8.5 \pm 0.8 \%$, respectively, $p<0.0001$ ).

\section{DISCUSSION}

The quality of T2DM care is affected not only by patient characteristics such as age, sex, ethnicity, socioeconomic position, educational status, and lifestyle but also by healthcare system factors such as healthcare organization, insurance system, financial incentives, clinical guidelines, and care-provider characteristics such as age, sex, and specialty [11-14]. The role of general practice and diabetic clinics in the management of diabetes is still a matter of debate. Studies have consistently shown that specialist care is associated with better process outcomes in type 1 diabetes [15]. For type 2 diabetes, some studies have suggested that patients with diabetes achieve better glycemic control with specialist care than with care by general practitioners $[11,16]$. Conversely, some studies, including our previous study $[6,16]$, have shown that there are no substantial differences between specialists and general practitioners in terms of outcome, although specialists tend to perform better than general practitioners in process measures $[11,17]$.

From our survey, overall HbA1c levels in patients with T2DM treated by general practitioners were lower than those in patients treated by specialists. In addition, the median HbA1c levels of patients treated by general practitioners in any group that was divided on the basis of patient age and BMI were lower than those of patients treated by specialists. However, multivariable regression analysis showed that lower age, higher BMI, and being female were associated with higher HbA1c levels in this study. Consistent with the findings of our previous study [6], the HbA1c levels of patients treated by general practitioners were lower than those of patients treated by specialists, regardless of patient age and BMI.

One possible explanation for this finding is that the proportion of therapy types differed between general practitioners and specialists. A higher proportion of patients treated by general

Table 7 Reasons for which general practitioners wish to consult and specialists prefer to be consulted

\begin{tabular}{llcc}
\hline Reasons & $\begin{array}{l}\text { General practitioners } \\
\boldsymbol{n}(\%)\end{array}$ & $\begin{array}{l}\text { Specialists } \\
\boldsymbol{n}(\%)\end{array}$ & $\boldsymbol{p}$ \\
\hline Higher HbAlc & $284(77.6 \%)$ & $60(89.6 \%)$ & 0.026 \\
Polypharmacy & $166(48.4 \%)$ & $51(78.5 \%)$ & $<0.0001$ \\
Initiation of insulin (basal insulin) & $209(58.4 \%)$ & $53(80.3 \%)$ & 0.001 \\
Adjusted insulin dosage (basal insulin) & $66(19.4 \%)$ & $32(49.2 \%)$ & $<0.0001$ \\
Initiation of insulin (multiple injection) & $295(81.9 \%)$ & $55(83.3 \%)$ & 0.786 \\
Adjusted insulin dosage (basal insulin) & $106(30.7 \%)$ & $38(58.5 \%)$ & $<0.0001$ \\
Initiation of GLP-1 RA & $197(56.8 \%)$ & $44(66.7 \%)$ & 0.135 \\
Adjusted GLP-1 RA dosage & $110(33.2 \%)$ & $25(37.9 \%)$ & 0.467 \\
\hline
\end{tabular}

$G L P-1 R A$ glucagon-like peptide 1 receptor antagonists 
practitioners than those treated by specialists received OAD therapy, whereas a higher proportion of patients treated by specialists than those treated by general practitioners received insulin therapy. Patients who received insulin therapy had higher HbA1c levels than those who did not receive insulin therapy, regardless of the care provider. The HbA1c levels of patients receiving OAD therapy from general practitioners were lower than those of patients receiving OAD therapy from specialists. General practitioners prescribed fewer OADs than did specialists, and HbA1c levels were found to be associated with the number of OADs prescribed, according to multivariable regression analyses. Our results suggest that specialists may treat patients who have a greater difficulty in achieving glycemic control and need to undergo intensive treatments such as insulin therapy or multiple combined OAD therapy.

General practitioners were more reluctant to intensify treatment for patients with T2DM, e.g., by initiating insulin or drug intensification, and specialists were less prone to clinical inertia than were primary care practitioners $[13,16]$. Under these circumstances, the appropriate response of general practitioners to elevated HbA1c levels would be to consult with a specialist. Accessibility to specialists may be a critical factor for smooth consultation, and the network between general practitioners and specialists may contribute to appropriate diabetes care. In this study, $88.2 \%$ of general practitioners indicated that it was easy to consult with specialists. Further, a shared care program between a specialized outpatient clinic and primary healthcare physicians has been shown to be non-inferior to management in a specialized outpatient clinic [18]. Another study has shown that training and continuous communication between primary care physicians and endocrinologists resulted in improvements in metabolic control among patients with diabetes and vascular disease in a primary setting [19]. In Italy, althpigh diabetic specialists have been endeavored for improving diabetic care $[20,21]$, for further improvement, a recent ongoing study is examining whether integrated care by general practitioners, diabetologists, and cardiologists can afford better outcomes in a population at-high-risk patients with diabetes [22]. Our study suggests that general practitioners may consult with specialists in a timely manner at the step of therapy intensification, such as the addition of a fourth OAD or more than four OADs or at the initiation of insulin therapy. In our study, the HbA1c level that general practitioners wished to consult with specialists about was higher than that for which specialists preferred to be consulted. In concordance with the findings of these reports, the network between general practitioners and specialists is expected to be bidirectional. In other words, not only should general practitioners consult with specialists but specialists should also inform general practitioners of guidelines and provide them with knowledge on new drugs as well as methods for initiation of insulin.

Weight control in patients with T2DM is a concern for physicians because BMI is associated with HbA1c values [3]. In our study, the BMIs of patients with T2DM cared for by general practitioners were found to be higher than those of patients cared for by specialists, especially among those with higher HbA1c levels. For weight management, lifestyle interventions, such as medical nutrition therapy, physical activity, and self-management education, and psychological support are necessary [23]. Many co-medical staff members need to participate in the education and empowerment of patients to introduce lifestyle changes. However, these collaborations may be difficult among general practitioners. Even among patients with T2DM and a good glycemic control status, such as HbA1c levels $<7.0 \%$, the BMI of patients cared for by specialists was lower than that of those cared for by general practitioners. This suggests that specialists may conduct the weight control in all patients with T2DM, regardless of their HbA1c levels. The association between BMI and all-cause mortality among patients with T2DM shows a U-shaped curve, and both lean and obese patients show an increased risk for allcause mortality [24]. Further, the Jichi Medical School Cohort Study in Japan showed that the risk of all-cause death in elderly patients with diabetes was substantially higher than that in lean patients of all ages, whereas the risk of all- 
cause death in obese patients was restricted to patients aged $<65$ years [25]. According to these reports, the weight management of patients with T2DM cared for by general practitioners was acceptable, except for those with high HbA1c levels.

Hypertension is common among patients with diabetes. Furthermore, it is a strong and modifiable risk factor for macrovascular and microvascular complications of diabetes [5]. Meta-analysis of clinical trials revealed that antihypertensive treatment of populations with diabetes and baseline blood pressure $<140$ / $90 \mathrm{mmHg}$ reduces the risk of atherosclerotic cardiovascular disease (ASCVD), heart failure, retinopathy, and albuminuria [26-28]. Therefore, most patients with diabetes and hypertension should be treated maintaining a target blood pressure of at least $<140 / 90 \mathrm{mmHg}$. A target blood pressure lower than $<149$ / $90 \mathrm{mmHg}$ may be beneficial for selected patients with diabetes. However, the JDS and the Japanese Society of Hypertension recommend that the target level of office blood pressure in patients with diabetes be $<130 /$ $80 \mathrm{mmHg}$ and an ACEi/ARB be used as a firstline antihypertensive drug because of its organprotective and insulin-sensitizing properties $[29,30]$. An ACEi/ARB is also recommended as a first-line treatment for hypertension in patients with T2DM and albuminuria in a position statement from the American Diabetes Association [5]. The EUROASPIRE IV survey showed that only $54 \%$ of patients with diabetes achieved a target blood pressure $<140$ / $90 \mathrm{mmHg}$ [31]. Canada is another country whose guidelines have retained a target blood pressure $<130 / 80 \mathrm{mmHg}$, and the achievement rate was 36\% among patients with T2DM [32]. In concordance with these reports, the target blood pressure $(<140 / 90 \mathrm{mmHg})$ achievement rates of $73.2 \%$ and $73.3 \%$ for patients with T2DM cared for by general practitioners and specialists, respectively, in our study appear to be adequate and similar to the findings of a Canadian report $(<130 / 80 \mathrm{mmHg} ; 37.7 \%$ and $40.0 \%$, respectively). Our results that diabetes specialists prescribe ACEi/ARB more often than general practitioners also suggest that specialists may focus on albuminuria and may consider the prevention of renal function more than general practitioners.

The limitations of this study include the sample size and nature of the participants. We randomly selected approximately $15 \%$ of the JMDPIMC members for study eligibility, and $6.9 \%$ participated in this study. In addition, it is likely that the majority of practitioners who agreed to participate in this study had an interest in diabetes care, considering participation was voluntary. However, the ratio of specialists to general practitioners in this study was $16.8 \%$, which is close to the ratio of certified diabetologists to general practitioners in Japan. Further, we did not collect precise information about the patients, such as duration of diabetes and complications, because the method of data collection was administration of a questionnaire among the busy practitioners. This study was cross-sectional; therefore, we could not clarify how the quality of diabetic care of general practitioners or specialists could contribute to improve the HbA1c levels.

In summary, the median HbA1c level of all patients treated by general practitioners was slightly lower than that of patients treated by specialists in this study. This suggests that collaboration between general practitioners and specialists is appropriate for managing patients with diabetes in Japan. In addition, blood pressure control by general practitioners was adequate and weight control was acceptable, except in patients with high HbA1c levels. The present study clearly demonstrated that many patients with T2DM are appropriately cared for by general practitioners instead of diabetes specialists in Japan, although the number of diabetes specialists is insufficient to cover all patients with diabetes. Finally, to match the constant increase in demand for diabetes care, provision of adequate quality of care by general practitioners is a solution to decrease the complications of diabetes and improve the quality of life of patients with diabetes. Our investigation may contribute to the policy of public health services in every country. 


\section{ACKNOWLEDGEMENTS}

The authors thank the clinics and hospitals that participated in this study.

Funding. This study was supported by a grant from the Kanagawa Association of Medical and Dental Practitioners. No funding or sponsorship was received for the publication of this study.

Editorial Assistance and Other Assistance. We would like to thank Mr. Takuma Katsumata and Ms. Mika Okayama for their statistical assistance. In addition, we would like to thank Editage (https://www.editage.com) for English language editing.

Authorship. All authors meet the International Committee of Medical Journal Editors (ICMJE) criteria for authorship for this article, take responsibility for the integrity of the work as a whole, and have given their approval for the publication of this version of the manuscript.

Disclosures. Shin-ichiro Shirabe received honoraria for lectures from Novo Nordisk and Sanofi-Aventis, and Yoko Matsuzawa received honoraria for lectures from Takeda. The remaining authors Keiko Arai, Tetsuo Nishikawa, Shohei Yuasa, Shigeyuki Ohtsu, Koich Hirao and Hisao Mori have nothing to disclose.

Compliance with Ethics Guidelines. The present study was approved by the ethics committee of the Kanagawa Association of Medical and Dental Practitioners, which includes lawyers and ethical experts, on May 1, 2018. The study approval number was 17007 . Informed consent was obtained from all enrolled patients at each clinic, in accordance with the Guidelines for Epidemiological Study of the Ministry of Health, Labour and Welfare of Japan.

Data Availability. The datasets generated during and/or analyzed during the current study are available from the corresponding author on reasonable request.
Open Access. This article is licensed under a Creative Commons Attribution-NonCommercial 4.0 International License, which permits any non-commercial use, sharing, adaptation, distribution and reproduction in any medium or format, as long as you give appropriate credit to the original author(s) and the source, provide a link to the Creative Commons licence, and indicate if changes were made. The images or other third party material in this article are included in the article's Creative Commons licence, unless indicated otherwise in a credit line to the material. If material is not included in the article's Creative Commons licence and your intended use is not permitted by statutory regulation or exceeds the permitted use, you will need to obtain permission directly from the copyright holder. To view a copy of this licence, visit http://creativecommons.org/licenses/by$\mathrm{nc} / 4.0 /$.

\section{REFERENCES}

1. Ministry of Health, Labour and Welfare, Japan, Annual Health, Labour and Welfare Report. 2017.

2. The Diabetes Control and Complication Trial Research Group. The effect of intensive treatment of diabetes on the development and progression of long-term complications in insulin-dependent diabetes mellitus. N Engl J Med. 1993;329:977-86.

3. Hamdy O, Mottalib A, Moris A, et al. Long-term effect of intensive lifestyle intervention on cardiovascular risk factors in patients with diabetes in real-world clinical practice: 5-year longitudinal study. BML Open Diabetes Res Care. 2017;5: e000259.

4. Japan Diabetes Clinical Data Management Study Group. http://jddm.jp/data/index-2018/.

5. De Boer IH, Bangalore S, Benetos A, et al. Diabetes and hypertension: a position statement by the Americam Diabetes Association. Diabetes Care. 2017;40:1273-84.

6. Arai K, Hirao K, Matsuba I, et al. The status of glycemic control by general practitioners and specialists for diabetes in Japan: a cross-sectional survey of 15,652 patients with diabetes mellitus. Diabetes Res Clin Pract. 2009;83:379-401. 
7. Haneda M, Ito H, Japan Diabetes Society (JDS)/Japan Geriatric Society (JGS) Joint Committee on Improving Care for Elderly Patients with Diabetes. Glycemic targets for elderly patients with diabetes. Diabetol Int. 2016;7:331-3.

8. Oishi M, Yamazaki K, Okuguchi F, et al. Change in oral antidiabetic prescriptions and improved glycemic control during the years 2002-2011 in Japan (JDDM32). J Diabetes Invest. 2014;5:581-7.

9. Kuzuya T, Nakagawa S, Satoh J, et al. The Committee of the Japan Diabetes Society on the diagnostic criteria of diabetes mellitus. Report of the Committee on the classification and diagnostic criteria of diabetes mellitus. Diab Res Clin Pract. 2002;55:65-85.

10. Alberti KG, Zimmet PZ. Definition, diagnosis and classification of diabetes mellitus and its complications. Part 1: Diagnosis and classification of diabetes provisional report of a WHO consultation. Diab Med. 1998;15:539-53.

11. De Berardis G, Pellegrini F, Francisosi M, et al. Quality of care and outcomes in type 2 diabetic patients: a comparison between general practice and diabetes clinics. Diabetes Care. 2004;27: 398-406.

12. Worswick J, Wayne SA, Bennett R, et al. Improving quality of care for persons with diabetes: an overview of systematic reviews-what does the evidence tell us? Syst Rev. 2013;2:26-39.

13. McBrien KA, Naugler C, Ivers $\mathrm{N}$, et al. Barriers to care in patients with diabetes and poor glycemic control-a cross-sectional survey. PLoS One. 2017;12:e0176136.

14. Tran AT, Bakke A, Berg TJ, et al. Are general practitioners characteristics associated with the quality of type 2 diabetes care in general practice? Results from the Norwegian ROSA4 study from 2014. Scand J Prim Health Care. 2018;36:170-9.

15. Cobin RH. Subspecialist care improves diabetes outcome. Diabetes Care. 2002;25:1654-6.

16. Shah BR, Hux JE, Laupacis A, Zinman B, von Walraven $C$. Clinical inertia in response to inadequate glycemic control. Do specialists differ from primary care physicians? Diabetes Care. 2005;28:600-6.

17. Greenfield S, Kaplan SH, Kahn R, Ninomiya J, Griffith JL. Profiling care provided by different groups of physicians: effect of patient case-mix (bias) and physician-level clustering on quality assessment results. Ann Intern Med. 2002;136: 111-21.
18. Munch L, Bennich BB, Overgaard D, et al. Management of people with type 2 diabetes shared between a specialized outpatient clinic and primary health care is non-inferior to management in a specialized outpatient clinic: a randomized, noninferiority trial. Diabet Med. 2019;36:854-61.

19. Duran A, Runkle I, Matia P, et al. Family physician and endocrinologist coordination as the basis for diabetes care in clinical practice. BMC Endocrine Disord. 2008;8:9-17.

20. Group di studio ANNALI AMD. AMD Annals: a model of continuous monitoring and improvement of the quality of diabetes care. Epidemiol Prev. $2011 ; 35: 18-26$

21. Rossi MC, Candido R, Ceriello A, et al. Trends over 8 years in quality of diabetes care: results of the AMD Annals continuous quality improvement initiative. Acra Diabetol. 2015;52:557-71.

22. Comaschi M, Di Lenarda A, Medea G, et al. INtegration of care for reaching targetS In Diabetic patiEnts: design of the INSIDE Study. Diabetes Ther. 2020;11:359-67.

23. Davies MJ, D'Alessio DA, Fradkin J, et al. Management of hyperglycemia in type 2 diabetes, 2018. A consensus report by the American Diabetes Association (ADA) and the European Association for the Study of Diabetes (EASD). Diabetes Care. 2018;41: 2669-701.

24. Kwon Y, Kim HJ, Park S, Park YG, Cho KH. Body mass index-related mortality in patients with type 2 diabetes and heterogeneity in obesity paradox studies: a dose-response meta-analysis. PLoS One. 2017;12:e0168247.

25. Yano $\mathrm{Y}$, Kario $\mathrm{K}$, Ishikawa $\mathrm{S}$, et al. Association between diabetes, leanness, and the risk of death in the Japanese general population. Diabetes Care. 2013;36:1186-92.

26. Emdin CA, Rahimi K, Neal B, Callender T, Percovic $\mathrm{V}$, Patel A. Blood pressure lowering in type 2 diabetes: a systematic review and meta-analysis. JAMA. 2015;313:603-15.

27. Ettehad D, Emdin CA, Kiran A, et al. Blood pressure lowering for prevention of cardiovascular disease and death: systematic review and meta-analysis. Lancet. 2016;387:957-67.

28. Brunstrom M, Calberg B. Effect of antihypertensive treatment at different blood pressure levels in patients with diabetes mellitus: systematic review and meta-analysis. BML. 2016;352:i717. 
29. Haneda M, Noda M, Origasa $H$, et al. Japanese clinical practice guideline for diabetes 2016. J Diabetes Investig. 2018;9:657-97.

30. Kai H. Blood pressure management on patients with type 2 diabetes mellitus. Hypertens Res. 2017;40:721-9.

31. Gyberg V, De Bacquer D, De Backer G, et al. Patients with coronary artery disease and diabetes need improved management: a report from the
EUROASPIRE IV survey: a registry from the EuroObservational Research Programme of the European Society of Cardiology. Cardiovasc Diabetol. 2015;14:133.

32. Grenier J, Goodman SG, Leiter LA, et al. Blood pressure management in adults with type 2 diabetes: insight from the diabetes mellitus status in Canada (DM-SCAN) survey. Can J Diabetes. 2018;42:130-7. 